\title{
Ontology to Improve CBIR System
}

\author{
Ashwini D. Gudewar \\ Department of Computer Engineering \\ Ramrao Adik Institute of Technology \\ Navi Mumbai, India
}

\author{
Leena R. Ragha \\ Department of Computer Engineering \\ Ramrao Adik Institute of Technology \\ Navi Mumbai, India
}

\begin{abstract}
The key problem in achieving efficient and user friendly Content Based Image Retrieval (CBIR), in domain of images is the development of a search mechanism to guarantee delivery of minimal irrelevant information (high precision) while insuring that relevant information is not overlooked (high recall). The current CBIR results need to be improved by indexing images according to semantics rather than objects that appear in the images. This problem of creating a meaning based index structure is solved using a concept based model with domain dependent ontology. The research analysis shows that, CBIR with ontology is still in primitive stage with very few topological relations exploited in the research, and the results still not satisfactory. Thus we propose a system for image retrieval which will use spatial information to build many of the topological relations like connectivity, adjacency, membership and orientation using ontology along with low level color and texture features for CBIR recognition.
\end{abstract}

\section{Keywords}

Image Retrieval; Content Based Image Retrieval (CBIR) System; Ontology; Spatial Information; Topological Relationship

\section{INTRODUCTION}

An image retrieval system is a computer system for browsing, searching and retrieving images from a large database of digital images. Most traditional and common methods of image retrieval utilize some method of adding metadata such as captioning, keywords, or descriptions to the images so that retrieval can be performed over the annotation words.

Interest in the potential of digital images has increased enormously over the last few years. Users in many professional fields like medical, engineering, architecture are exploiting the opportunities offered by the ability to access and manipulate remotely-stored images in all kinds of new and exciting ways. However, it is also discovered that the process of locating a desired image in a large and varied collection can be a source of considerable frustration. While it is perfectly feasible to identify a desired image from a small collection simply by browsing and more effective techniques are needed with collections containing thousands of items. The problems of image retrieval are becoming widely recognized, and the search for solutions an increasingly active area for research and development.

Image Retrieval Systems are broadly categorized into Textbased image retrieval and Content based image retrieval.

\section{Text Based Image Retrieval System}

In Text Based Image Retrieval system, image search is supported by augmenting images with keyword-based annotations and the search process always relies on keyword matching techniques. Text Based Image Retrieval system is lexically motivated rather than conceptually motivated, which leads to irrelevant search results in information retrieval.
Limitations of Text based Image Retrieval:

- Manual annotations require too much time and are expensive to implement.

- The contents of medical images are difficult to be concretely described in words. For example, irregular organic shapes cannot easily be expressed in textual form.

- Users should have complete domain knowledge in order to formulate appropriate keywords for a valid query.

- Additionally, the difficulty in dealing with non-visual objects like expressing feelings or emotions.

Thus to overcome these limitations of text based image retrieval, content based image retrieval methods are proposed.

\section{Content-based Image Retrieval}

Problems with traditional methods of image indexing have led to the rise of interest in techniques for retrieving images on the basis of automatically-derived features such as color, texture and shape - a technology now generally referred to as Content-Based Image Retrieval (CBIR). Content-based image retrieval is an alternative and complement to traditional text based image searching. Content based image retrieval means that the search will analyze actual contents of the image by using image analysis technique. The term content in this context refers to properties of the image called low level features such as color, shape, texture, or any other information that can be derived from the image itself. These systems employ image processing technologies to extract visual features and then similarity measurements are applied to get the relevant images. Feature extraction algorithms extract features and store them in the form of multidimensional vectors. Afterwards, similarity or dissimilarity measurement between two feature vectors is defined for each feature.

Limitations of Content based Image Retrieval:

- Semantic gap, the discrepancy between the low level features that are automatically extracted by machine and the high level concepts of human vision and image understanding.

- In CBIR we generally have large database which contains images with varying huge sizes, so CBIR of extracting relevant images is a very time consuming process.

- Low retrieval precision together with the requirement of advanced image processing and pattern recognition techniques.

Hence to overcome the limitations of these image retrieval systems we proposed a system based on spatial ontology to remove the semantic gap and using this ontology tag the categorization of images reduces the huge database search. Since the categorization helps to gather relevant images to certain extent the fines feature extraction using CBIR may improve upon the retrieval precision. Hence in this paper, we propose to build ontology based CBIR. 


\subsection{Ontology based Image Retrieval}

The term "ontology" can be defined as a formal explicit specification of shared conceptualization. Conceptualization refers to an abstract model of some phenomenon in the world which identifies the relevant concepts of that phenomenon. Explicit means that the type of concepts used and the constraints on their use are explicitly defined. Formal refers to the fact that the ontology should be machine readable.

Ontology typically contains a hierarchy of concepts within a domain and describes each concept's crucial properties through an attribute value mechanism. Ontologies were developed in artificial intelligence to facilitate knowledge sharing and reuse. Ontologies define the concepts and relationships used to describe and represent an area of knowledge. Ontology provides the ability to model the semantics of what occurs in images such as objects, events etc. Ontology also provides an elegant mechanism to formally organize image content in small, logically contained groups (ontological concepts). Ontology enables ontological concepts to be linked, merged and distinguished with other concepts in logically contained groups. It provides a formalized common understanding on a specific domain between humans and computers. Hence ontology represents the knowledge in a hierarchical structure which is used to describe, organize image collection and it also shows the relation between these images.

Ontology based Image Retrieval uses knowledge representation which combines the features of text based image retrieval and content based image retrieval. The main purpose of ontology is to represent an image in semantic manner. Hence the image is represented in machine understandable manner, this leads to retrieval task easier.

The organization of this paper is as follows. Literature Survey is given in section 2. The proposal and proposed system is explained in detail in section 3. Analysis of the proposed system with one case study and RDF schema is given in section 4. Section 5 concludes this paper.

\section{LITERATURE SURVEY}

When we want to search any specific image into the database, image queries are characterized into three levels of abstraction: primitive features such as color or shape, logical features such as the identity of objects shown and abstract attributes such as the significance of the scenes depicted. Current indexing practice for images relies largely on text descriptors or classification codes. CBIR operates on a totally different principle from keyword indexing. Primitive features characterizing image content, such as color, texture and shape are computed for both stored and query images, and used to identify top K most closely matching stored images.

CBIR systems currently operate effectively only at the lowest primitive feature levels and most users demand higher levels of retrieval. None of the systems can search effectively for, say, a photo of a dog - though some semantic queries can be handled by specifying them in terms of primitives. A beach scene, for example, can be retrieved by specifying large areas of sky blue at the top of the image, and some land color shade at the bottom. There is evidence that combining primitive image features with text keywords can overcome some of these problems, though little is known about how such features can best be combined for retrieval.

In [2], to extract the selected features based on color and to index the database images like food, flowers etc. Global Color Histogram $(\mathrm{GCH})$ was used. The similarity between two colors given by indices can be measured by the Euclidean distance between the color points in the HSV color space. Though this system showed good precision value but recall value is poor.

In [3] texture representation method for image retrieval based on Grey Level Co-occurrence Matrix (GLCM) is proposed. GLCM expresses the texture feature according to the correlation of the couple pixels gray level values at different positions. The texture features such as entropy, energy, contrast and homogeneity can be extracted from the cooccurrence matrix of gray levels of an image. The experimental results showed that the proposed method yields higher precision and recall with reduced vector dimension along with some of the irrelevant images.

In [4], the image features like global color, texture and shape are considered for scene image retrieval. For color feature extraction, color histograms such as Local Color Histogram $(\mathrm{LCH})$, Global Color Histogram $(\mathrm{GCH})$ and Fuzzy Color Histogram (FCH) are used. For extracting textures Statistical, Structural, Spectral approaches are used. In addition to this, Tammura Texture and Wavelet Transform are used. These extracted features will be forwarded to Feature Vector Module. The Direct Euclidian Distance between an image from database and query image is calculated for similarity measures. Comparison based on color feature gives 4 relevant images while texture based gives 3 relevant images out of 10 images.

The objective of [5] is to develop a technique which captures color and texture descriptors of an image, and has a shape descriptor in terms of invariant moments computed on the edge image. This proposed CBIR system is based on Dominant color and Grey Level Co-occurrence Matrix (GLCM) texture and shape which are used to describe image from the different aspects for more detailed information in order to obtain better search results. This method is applied on set of dinosaur images. Precision observed was very low, only 7 images were matching with the given query image.

A novel hybrid two stage universal CBIR technique using both color and texture features extraction is proposed in [6]. In the first stage for color feature extraction, color moments up to the fourth order are extracted and are used in deriving the respective histograms which forms the color feature vector. In the second stage for the texture feature extraction the CCM (Color Co-occurrence Matrix) technique employed takes into account the correlation between the RGB color bands in all the eight directions while computing the texture features. In every stage the distance between the query image and the image in the database is calculated by using relative distance measure. The resultant distance between the query image and the image in the database is calculated by using a weighted distance classifier. Thus, a hybrid fusion method is achieved that has better performance than other color-spatial based methods and promises to give more relevant output to the user.

The resultant output shown in [2][3][4][5] and [6] contains the irrelevant images which degrades systems performance and results in less precision and recall value. Hence to get proper results an efficient image retrieval system is required to be designed. The main limitation of CBIR system is semantic gap, to bridge this gap we have to provide some kind of knowledge or intelligence to the system as per the user's requirement and some robust methodologies are also required. To have truly intelligent systems, knowledge needs to be captured, processed, reused and communicated. All these 
tasks can be achieved using ontologies. Hence if we design ontology based image retrieval system with CBIR then it improves the recall and precision of the system.

Some of the papers detail which has used ontology for image retrieval is:

Current research on shape based classification has been generally aimed at utilizing various visual features. Previous research has shown that the existing knowledge in a specific domain can assist in understanding the image content. In [7] a shape ontology framework for bird classification is proposed. This framework creates domain and shape ontology. These two ontologies describe bird images in different aspects and both are mapped to each other, so that the textual domain and visual perception knowledge can be integrated together for the assistance of bird classification. Here four different bird shapes, that is, duck, penguin, tree-creeper, and fairy-wren like shapes and 105 bird images are collected. Each shape category contains $25,28,28$ and 24 images respectively.

Table 1: Precision and recall results

\begin{tabular}{|c|c|c|c|c|}
\hline & Duck like shape & Penguin & Fairy-wren & Tree-creeper \\
\hline Precision & 0.607 & 0.875 & 0.613 & 0.455 \\
\hline Recall & 0.68 & 0.75 & 0.679 & 0.417 \\
\hline
\end{tabular}

In [8] novel approach to image retrieval, which associates the use of an ontology and of low level picture descriptors. The application specified in this paper uses the high level semantics of an inheritance system to control the retrieval process and to propose browsing variants to the user. CBIR applications do not offer enough interaction means to the user and that they are not self-explanatory. Thus RetrievOnto system is designed to cope with these problems and provide as easy to use user interface.

In [9], the object ontology is used to allow the qualitative definition of the high-level concepts the user queries for an image. Using the intermediate-level descriptors clearly irrelevant image regions is rejected. A relevance feedback mechanism employing the low-level features is invoked to produce the final query results. Here search is done for "red car" as query image and results in displaying first top 20 images that satisfies the conditions, out of these only 12 images are matching with query image.

In [10] an ontology based CBIR strategy is proposed which narrows the semantic gap when retrieving images. A Selforganizing feature map (SOM) based quantization method is used to categorize the objects that are the basis for the semantic ontology. The proposed image retrieval model is based on a concept hierarchy. Multiple features automatically extracted from the segmented regions in images are classified into various categories using a fuzzy SOM. Moreover, all the regions of images within a category are further clustered into several sub-classes. Images are retrieved by searching through a single major category and then different sub-classes. This approach overcomes the shortcomings of image-to-image matching. Multiple semantic image retrieval can be fulfilled by simultaneously considering both global and local features.

\subsection{Literature Survey Analysis}

There are hundreds of millions of images of mixed category available on the current World Wide Web. So searching desired image from such large database is very difficult. Initially we used to search an image based on the text associated with the image (image name). But this requires lots of time for manual annotations. In order to improve the manual search, researchers worked on to CBIR techniques to improve the image retrieval. In CBIR images are retrieved by comparing primitive features such as color, texture, shape etc. But again there are some problems with this technique. We can use color histograms for comparing two images but they do not provide semantic information about the image. With CBIR technique, there is a large semantic gap due to differences between the information extracted from the visual features of images and the interpretation made by users which needs to be bridged. This semantic gap if filled, the CBIR results can be improved.

Hence, to bridge this semantic gap we can use ontology. Ontology provides the way to organize the web information in structured way. The ontology based image retrieval uses knowledge representation which combines the features of text based image retrieval and content based image retrieval. The main purpose of ontology is to represent an image in semantic manner. Hence the image is represented in machine understandable manner, this leads to retrieval task easier.

The research analysis shows that, CBIR with ontology is still in primitive stage, and the research results still show extraction of some of the irrelevant images. We can still improve any image retrieval system if recall value of the system is increased. Based on these analyses, we propose an ontology based CBIR solution.

\section{PROPOSED SYSTEM}

In classical CBIR system images are retrieved based on low level features such as color, texture and shape. In CBIR system generally features based on color are extracted from the images and then these features are matched with the images stored in the database. But in this system there are chances that we can get irrelevant images also. Thus to overcome this drawback of CBIR system and to bridge the semantic gap between users and machines one system is proposed which uses spatial domain ontology. Semantic gap is the lack of coincidence between the information that one can extract from the visual data and the interpreting that the same data have for user in a given situation.

In this proposed system segmentation is performed to divide an image into several regions. Then spatial domain ontology is formed using topological relationship and all the images which are stored in the database are compared with the query image. This comparison results in getting first level output which consist of most of the relevant images, removing irrelevant images from the database and this database is now called as the refined database. For further operation CBIR technique is used where will extract the color features of the images stored in the refined database and then matching operation is performed with query image.

Generally, to improve the search results of CBIR technique ontology is used. But we are proposing a system which will create spatial domain ontology using high level features along with CBIR approach which is based on low level feature.

\subsection{Spatial Domain Ontology}

Sometimes the ontology-based image retrieval system does not represent the contents about the image properly. There are various objects in the image and it needs to describe objects with spatial relationships for more correctly representing the images.

Regions or objects with similar color and texture properties can be easily distinguished by imposing spatial constraints. 
For instance, regions of blue sky and ocean may have similar color histograms, but their spatial locations in images are different. Therefore, the spatial location of regions (or objects) or the spatial relationship between multiple regions (or objects) in an image is very useful for searching images.

The general classes in this ontology include:

- Domain (D): A domain is defined as an area of interest or knowledge. In the ontology, the domain indicates the context with which the specific knowledge is associated. For example, birds, animals and nature scene images.

- Concept (C): A concept is a generic idea derived from specific instances. Concepts are required to explain all things that have specific meaning in the scope of human visual perception. Concept can be represented as an aggregation of several objects. Consider sky as an example which normally contains birds, clouds etc, so we can say sky is one concept.

- Object (O): An object is defined as the minimum unit of meaningful things in a specific domain. Objects are parts of given image, for example in case of car image, car is one object, road is another object etc.

- Features (F): This represents low level features like color, texture and shape of an object in the images.

- ConstraintRules (Cs): To describe the object from the human visual perception, some conditions and restrictions are added to the ontology, which are called ConstraintRules.

The general properties in ontology include:

- containConcepts (CC): This property is utilized to indicate what concepts are involved in a domain.

- musthaveObjects (MSH): This property indicates a strong relationship between concepts and objects. The objects associated to the concepts using this property can be understood as the key-objects for the concepts.

- mayhaveObjects (MAH): This property indicates a weak or optional relationship between concepts and objects. mayhaveObjects is setup to approach the user preference and improve the accuracy of concept descriptions.

\subsection{Topological Relationship}

Topological relationships describe relationships between objects in space. By objects we mean natural or manmade features like lakes, trees, houses, roads, etc. Important topological relationships are explained below:

- Proximity describes how close or how far away two (or more) objects are - my school is near my house.

- Connectivity means how two objects are linked with each other - the downtown is connected with a subway.

- Adjacency explains whether two objects are next to each other or not - Tom's house is next to Pam's.

- Membership means whether an object belongs to a particular group or not - apple is a type of fruit.

- Orientation describes the location and direction of an object - the house is located 10 miles north of the city
In our system we are trying to identify four relationships that exist between two objects namely Adjacency, Connectivity, Membership and Orientation using following given topological relationships.

Table 2. Topological Relationships

\begin{tabular}{|c|l|}
\hline Property Name & \multicolumn{1}{|c|}{ Meaning } \\
\hline Disjoint & $\begin{array}{l}\text { Two objects do not share } \\
\text { either border or interior }\end{array}$ \\
\hline Meet & $\begin{array}{l}\text { Two objects share border but } \\
\text { not interiors }\end{array}$ \\
\hline Overlap & $\begin{array}{l}\text { Two objects overlap. This } \\
\text { means that the interior } \\
\text { overlap and the border cross }\end{array}$ \\
\hline Overlap_with_disjoint_border & $\begin{array}{l}\text { The interiors of two objects } \\
\text { overlap but the borders do } \\
\text { not }\end{array}$ \\
\hline Cover & $\begin{array}{l}\text { One object is inside the other } \\
\text { but shares a part of its border }\end{array}$ \\
\hline CoveredBy & $\begin{array}{l}\text { One object is inside the other } \\
\text { but do not shares part of its } \\
\text { border }\end{array}$ \\
\hline Inside & $\begin{array}{l}\text { One object is entirely inside } \\
\text { the other }\end{array}$ \\
\hline
\end{tabular}

Connectivity relationship is achieved using Disjoint and Meet property. Adjacency relationship is achieved using Overlap and Overlap_with_disjoint_border. Membership relationship is achieved using Cover, CoveredBy and Inside property. By finding the location of particular object in an image orientation relationship can also be achieved.

Since windows of different size can be used to divide an image into parts which are called as zones. These zones are used to represent proper position of particular object present in the image. If object is getting divided into more number of zones then to get fine details of an object, we can increase the window size. For example, generally we use $3 \mathrm{X} 3$ window to divide an image instead of that we can use $5 \times 5$, 9X9 window to extract fine details of an object.

In this proposed system we are dividing an image into different zones by applying window of size $3 \times 3$. Some naming conventions are used to represent particular zones where the required object is present (position), as shown below.

Table 3. Naming Convention for Orientation Relationship

\begin{tabular}{|c|c|c|}
\hline $\begin{array}{c}\text { TOP LEFT } \\
(\mathrm{TL})\end{array}$ & $\begin{array}{c}\text { TOP MIDDLE } \\
(\mathrm{TM})\end{array}$ & $\begin{array}{c}\text { TOP RIGHT } \\
(\mathrm{TR})\end{array}$ \\
\hline $\begin{array}{c}\text { MIDDLE } \\
\text { LEFT(ML) }\end{array}$ & $\begin{array}{c}\text { MIDDLE MIDDLE } \\
(\mathrm{MM})\end{array}$ & $\begin{array}{c}\text { MIDDLE RIGHT } \\
(\mathrm{MR})\end{array}$ \\
\hline $\begin{array}{c}\text { BOTTOM LEFT } \\
(\mathrm{BL})\end{array}$ & $\begin{array}{c}\text { BOTTOM MIDDLE } \\
(\mathrm{BM})\end{array}$ & $\begin{array}{c}\text { BOTTOM RIGHT } \\
(\mathrm{BR})\end{array}$ \\
\hline \multicolumn{2}{|c}{}
\end{tabular}

\subsection{Block Diagram of Proposed System}

The overall system is explained with block diagram as shown in figure 1. 


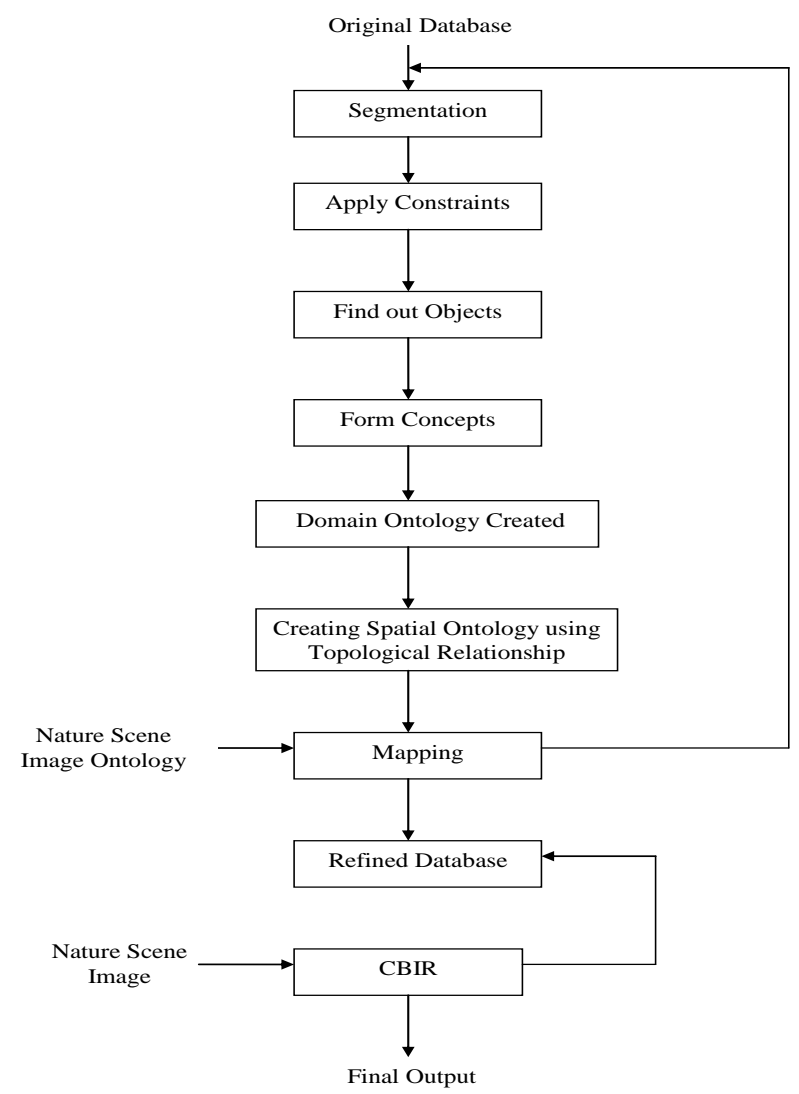

Fig 1: Proposed System

\subsection{Explanation of Proposed System}

Initially segmentation is applied on original database to get different regions of the image. This is based on color and border detection thus individual objects are detected. The general term which is used to represent group of objects is called as concept. For example consider "sky" as one object, many times we see birds flying in the sky, cloudy atmosphere and so on. So bird and clouds are the part of sky. Thus we can represent 'sky' as concept which consist of different objects like bird, cloud etc.

Domain is formed by combining objects and concepts together and this is called as domain ontology. After creating domain ontology, we will find out the spatial relationship between the objects to create spatial ontology. For this, topological relationship has to be generated between two objects which indicate how two objects are related to each other in terms of connectivity, direction, distance etc.

Mapping operation is performed to find out the relevant image stored in the database, by comparing individual image with the query image. If we get similarity measure computed using sum of the weighted differences of the feature values within some threshold value say ' $\mathrm{T}$ ' then we say that image is relevant image and these images will be stored in the refined database. Hence to improve the recall value we are trying to reduce the size of the database firstly by performing the ontology mapping of the image to be retrieved and the database images and then applying CBIR on this refined database to extract top $\mathrm{N}$ images. The classical CBIR extracts features based on moments, color, shape, texture etc. from the image to be retrieved and performs similarity measures with the features extracted from the database images to retrieve the most relevant top $\mathrm{N}$ matches with high precision and recall.

\section{ANALYSIS}

For any query image first we have to create domain ontology by finding different objects present in the image. On these objects properties are applied based on the human visual perception. With these properties we will decide which should be present in the image i.e. if the particular object is present in the image then only consider that image otherwise do not. How to build domain ontology along with spatial relationship for nature scene image is shown in figure 2.

Consider we want to search 'Nature Scene Images' stored in the database. The constraint rules are applied for extracting different regions of the images. For example consider that nature scene must contain water, sky, land but it may also contain trees, grass etc. In constraint user specifies the things which should be present in the image. These constraints are implemented using some properties as mentioned in table 1.

Extracting different regions from the image based on low level features to identify proper object and concept is nothing but creating domain ontology for particular image. After creating general domain ontology next job is to find spatial relationship between two objects. Consider following image for case study.

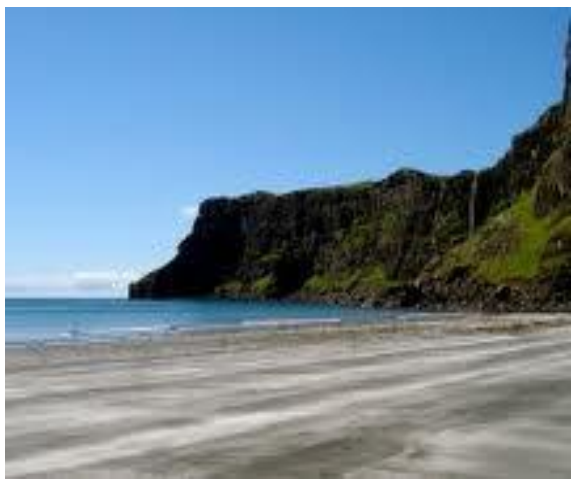

Fig 3: Nature Scene Image

Now will divide this image in different zones by applying window of size $3 \times 3$ to find position of individual object present in the image as shown below.

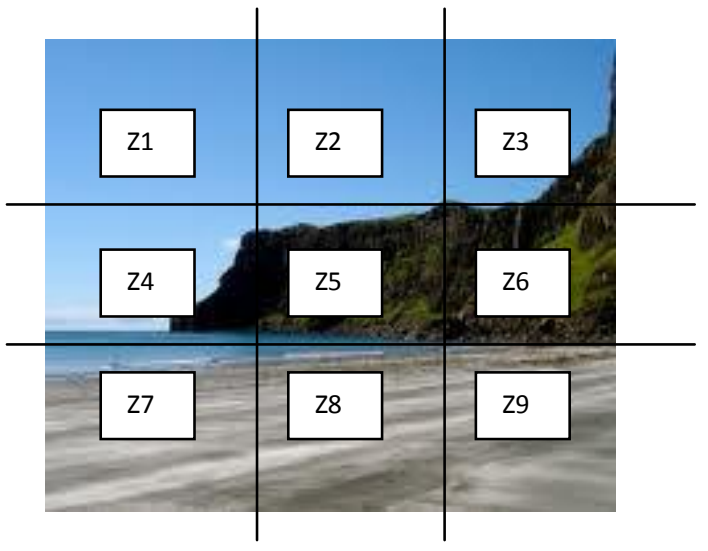

Fig 4: Modified Nature Scene Image 


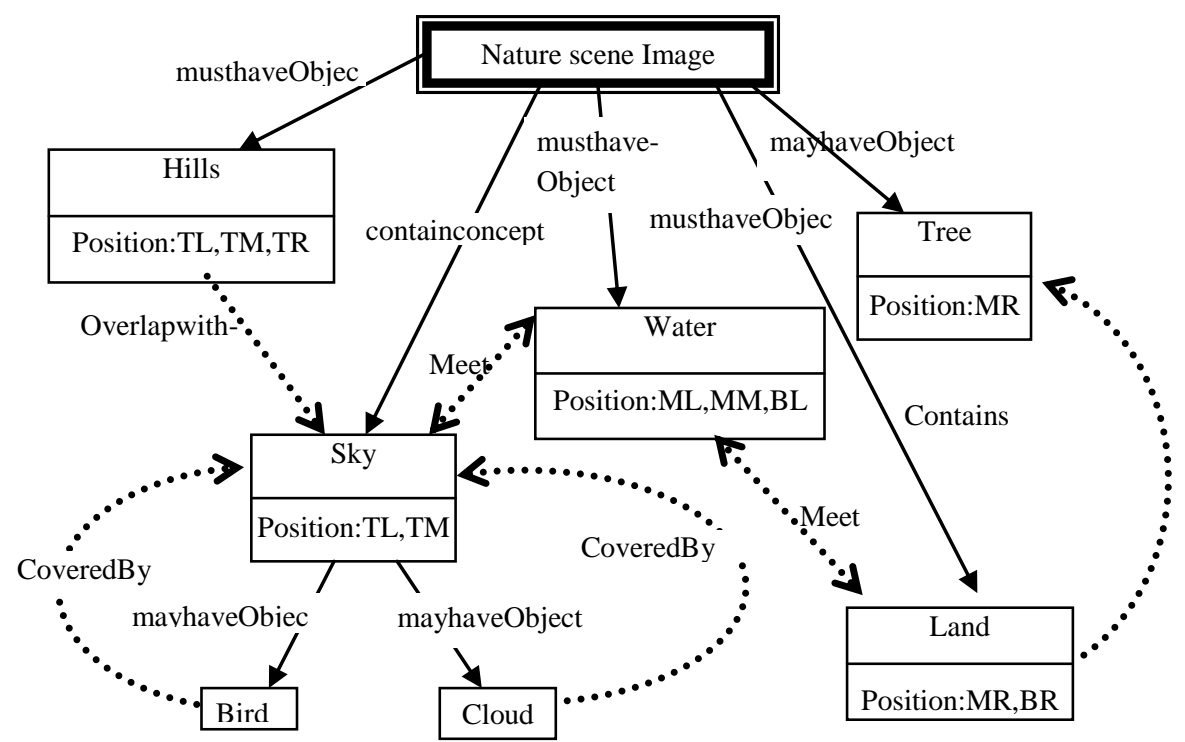

Fig 2: Ontology for Nature Scene Image

For example, sky is present in upper portion of the image so we can say sky is present in top most portion of the image and water must be present in middle portion of the image.

Here zone Z1 and Z2 clearly specify that this is the part of the sky and appears in top most area of the image, so along with the object representation we can specify the position of 'Sky' object as top left (TL) and top middle(TM) as mentioned in table 2. A ZONE Z7, Z8 and Z9 represent land area and its position is specifies as bottom left (BL), bottom middle (BM) and bottom right (BR).

After getting spatial information means the position of each and every object in the image will find out topological relationship between these objects.

For example, normally sky is seen behind the hills so we can say interior of the hills overlap with the sky. Another example, flowing water is always surrounded by some land, then we can say both share their boundary but not the interior. Thus connectivity, membership and adjacency such spatial relationship is achieved using some properties which are mentioned in the table 1 .

Based on the spatial relationships and their properties, we build the spatial ontology using the web ontology language (OWL) $[13,14]$ and RDF. Resource Description Framework (RDF) defines a simple model for describing relationships among resources in terms of named properties and values. RDF properties represent relationships between resources. As such, the RDF data model can therefore resemble an entity-relationship diagram.

All things described by RDF are called resources, and are members of the class, rdfs:Resource. The rdf:Class corresponds to the generic concept of a type or category of resource. The rdf:Property represents those resources that are RDF properties. The rdf:type property indicates that a resource is a member of a class. The rdfs:label property is used to provide a human-readable version of a resource's name.

We are mainly focusing on spatial information, so class name is SpatialRegion. Each object represents different regions present in the image and connections between these objects are represented using topological relationship like proximity, adjacency, membership and connectivity with some properties as mentioned in table 2 . The table 3 shows the spatial ontology written by OWL.

\section{Table 4. Spatial domain ontology}

<rdf:RDF xmlns:rdf="http://www.w3.org/1999/02/22-rdfsyntax-ns\#"

xmlns:rdfs="http://www.w3.org/2000/01/rdf-schema\#"

xmlns:owl="http://www.w3.org/2002/07/owl\#"

xmlns:xsd="http://www.w3.org/2001/XMLSchema\#"

Xmlns:sr="http://vector.chosun.ac.kr/spatial-basic\#">

$<$ rdfs:comment $>$ This ontology defines constructs for

expressing spatial relations </rdfs:comment $>$

$</$ owl:Ontology >

$<$ owl:Class rdf:ID="SpatialRegion">

$<$ rdfs:label $>$ SpatialRegion $</$ rdfs:label $>$

$</$ owl:Class $>$

<owl:ObjectProperty rdf:ID="Contains">

$<$ rdfs:label $>$ Contains $</$ rdfs:label $>$

$<$ rdfs:domain rdf:resource="\#SpatialRegion" />

$<$ rdfs:range rdf:resource="\#SpatialRegion" / >

<rdf:type

rdf:resource="http://www.w3.org/2002/07/owl\#ProximityPro

perty" />

$</$ owl:ObjectProperty>

<owl:ObjectProperty rdf:ID="Overlap">

<rdfs:label>Overlap</rdfs:label>

$<$ rdfs:domain rdf:resource="\#SpatialRegion" />

$<$ rdfs:range rdf:resource="\#SpatialRegion" />

$<$ rdf:type

rdf:resource="http://www.w3.org/2002/07/owl\#AdjacencyPro perty" />

$</$ owl:ObjectProperty>

<owl:ObjectProperty rdf:ID="CoveredBy">

$<$ rdfs:label $>$ CoveredBy $</$ rdfs:label $>$

$<$ rdfs:domain rdf:resource="\#SpatialRegion" />

$<$ rdfs:range rdf:resource="\#SpatialRegion" / >

$<$ rdf:type

rdf:resource="http://www.w3.org/2002/07/owl\#MembershipP roperty" $/>$

$</$ owl:ObjectProperty $>$ 
<owl:ObjectProperty rdf:ID="Meet">

$<$ rdfs:label $>$ Meet $</$ rdfs:label $>$

$\langle$ rdfs:domain rdf:resource="\#SpatialRegion" $/\rangle$

$<$ rdfs:range rdf:resource="\#SpatialRegion" / >

$<$ rdf:type

rdf:resource="http://www.w3.org/2002/07/owl\#ConnectivityP roperty" />

$</$ owl:ObjectProperty $>$

$</$ rdf:RDF $>$

\section{CONCLUSION}

Here we are trying to address the problem when an end-user is faced with a repository of images whose content is complicated and partly unknown to the user. Such situations occur frequently when using public image databases on the web. Considerable research has been done to propose effective mechanisms for image retrieval based on image content. Two major paradigms are: text-based metadata image retrieval and content-based image retrieval (CBIR)

In the text based approach, image retrieval is based on textual descriptions about images. The problem of text-based image retrieval is that it can sometimes provide too many redundant images or no image at all, text metadata is expensive to provide in terms of human effort, and alternative approaches are needed.

To overcome the limitations of the text-based approach, the use of the image visual contents has been proposed. This category of approaches utilizes the visual contents by extracting low level indexing features for each image or image segment (region). A major drawback of such approaches is that, in order to start a query, the availability of an appropriate key-image is assumed. Even assuming perfect feature extraction such systems are hard to use as there is a large gap between physical features and human visual perception and semantic concepts i.e. semantic gap.

Thus an ontology based CBIR strategy is proposed which narrows the semantic gap when retrieving images. In our proposed system first we are creating a spatial domain ontology using topological relationship and then classical CBIR is applied. This system helps to achieve following advantages:

1. With the use of ontology human requirements are conveyed in the process of extraction that means Semantic gap is removed.

2. In this proposed system first we are creating ontology which reduces the large database to smaller one. This means most of the irrelevant images are removed keeping only those which satisfy the ontology constraints and this updated database is used for further operation.

3. This system improves the recall (maximum relevant information) and precision (minimal irrelevant information) of the system. Since we are trying to remove the irrelevant images at first level output i.e. after applying ontology.

\section{REFERENCES}

[1] Ying Liu, Dengsheng Zhang, Guojun Lu, Wei Ying Ma, 2006, " A Survey of Content based Image Retrieval with High Level Semantics ", The journal of the Pattern Recognition Society, Page No. 262-282.
[2] Asmita Deshmukh, Gargi Phadke, 2011, "An Improved Content Based Image Retreival", International Conference on Computer \& Communication Technology (ICCCT).

[3] Asmita Deshmukh, Leena Ragha and Gargi Phadke, February 2012, "An Effective CBIR using Texture", IJCA Proceedings on International Conference and Workshop on Emerging Trends in Tchnology (ICWET).

[4] Swati S. Sakhare, Vrushali G. Nasre, 2011, “ Design of Feature Extraction in Content Based Image Retrieval (CBIR) using Color and Texture ", International Journal of Computer Science \& Informatics , Vol. I, Issue-II, Page No. 57-61.

[5] M. Babu Rao, Dr. B. Prabhakara Rao, Dr. A. Govardhan,2011, “ Content Based Image Retreival using Dominant Color, Texture \& Shape " , International journal of Engg. Science \& Technology , Vol.3, Page No. 2887-2896.

[6] Dipti Jadhav, Gargi Phadke, Dr Satish Devane, 2012, “ Colour and Texture Feature Based Hybrid Approach for Image Retrieval ", Advances in Intelligent and Soft Computing,SpringerLink.

[7] Yuee Liu, Jinglan Zhang, Dian, Shlomo Geva, 2007, “ A shape Ontology Framework for Bird Classification ", 9th Biennial Conference of the Australian Pattern Recognition Society on Digital Image Computing Techniques and Applications IEEE, Computer Society , Page No. 478 - 484.

[8] Adrian Popescu, Christophe Millet, Pierre Alain, July 2007, " Ontology Driven Content Based Image Retrieval ", ACM International conference on Image and Video Retrieval.

[9] Vasileios Mezaris, Ioannis kompatslaris and Michael G. Strintzis, 2003, “An Ontology Approach to ObjectBased Image Retrieval ", IEEE International Conference on image Processing, Vol. 3, Page No. 511-514.

[10] Tsun-Wei Chang, Yo-Ping Huang, Frode Eika Sandnes, 2008, “ An Ontology Oriented Region Based Image Retrieval Strategy ", IEEE International Conference on Systems, Man and Cybernetics, Page No. 2671 - 2676.

[11] Song Liu, Liang-Tien Chia and Syin Chan, 2004 " Ontology for Nature Scene Image Retrieval ", Springer-Verlag Berlin Heidelberg, Page No.1050-1061.

[12] Antonia Penta, Antonio Picariello, Letizia Tanca, 2007 “ Towards a definition of an Image Ontology ",18th International Conference on Database and Expert Systems Applications IEEE, Page No. 74 - 78.

[13] Hyunjang Kong, Pankoo Kim, Myungggwon Hwang, 2006 , "The Study on the semantic Image Retrieval based on the Personalized Ontology ", International Journal of Information Technology, Vol. 12, No. 2 , Page No. 35-46.

[14] Dieter Fensel, Frank van Harmelen and Ian Horrocks, 2011, " OIL : An Ontology Infrastructure for the Semantic web ”, IEEE Intelligent systems , Page No. 38 -45 .

[15] Preeti aggarwal, H.K.Sharma, Gagandeep jindal, April 2009,“ Content Based Medical Image Retrieval: Theory, Gaps and Future Directions ", ICGST International 
Journal on Graphics, Vision and Image Processing, GVIP ,Vol. 9, Issue II , Page No. 27-37.

[16] D. Brickley, R. Guha(eds.), 27 March 2000, Resource Description Framework(RDF) Schema Specification. W3C Candidate Recommendation, http://www.w3.org/TR/2000/CRrdf-schema-20000327.

[17] T. Berners-Lee, J. Hendler and O. Lassila, 2001, The Semantic Web. Scientific Am. , Page No. 34-43.

[18] Ian Horrocks, December 2008, “ Ontologies and the Semantic Web ", Communications of the ACM, vol. 51, no. 12
[19] ChunNian Liu, JunYing He, 2009, “ Application of Domain Ontology-based on Semantic Web Technology ”, IEEE Computer Society, Page No.133-136.

[20] Thomas M. Deserno, Sameer Antani and Rodney Long, 2007, “ Ontology of Gaps in Content-Based Image Retrieval ”, Journal of Digital Imaging.

[21] Workshop on the Application of Language and Semantic Technologies to support Knowledge Management Processes' at EKAW 2004.

[22] Darshak G. Thakore, A. I. Trivedi, “ Content based image retrieval techniques - Issues, analysis and the state of the art". 